\title{
Characteristics and outcomes of COVID-19 associated stroke: a UK multicentre case-control study
}

\author{
Richard J Perry (1) , 1,2 Craig J Smith (1) , 3,4 Christine Roffe (1), 5,6 \\ Robert Simister @ (1) ${ }^{1,2}$ Saravanan Narayanamoorthi, ${ }^{7}$ Richard Marigold (1), ${ }^{8}$ \\ Mark Willmot, ${ }^{9}$ Anand Dixit, ${ }^{10}$ Ahamad Hassan, ${ }^{11}$ Terence J Quinn (1), ${ }^{12,13}$ \\ Sandeep Ankolekar, ${ }^{14}$ Liqun Zhang, ${ }^{15}$ Soma Banerjee $\left(10,{ }^{16}\right.$ Urwah Ahmed, ${ }^{9}$ \\ Nishita Padmanabhan, ${ }^{6}$ Phillip Ferdinand, ${ }^{6}$ Frances McGrane, ${ }^{13}$ Azra Banaras, ${ }^{1}$ \\ Isobel H Marks, ${ }^{16}$ David J Werring (1) ${ }^{1,2}$ on behalf of the SETICOS collaborators
}

\begin{abstract}
- Additional material is published online only. To view please visit the journal online (http://dx.doi.org/10.1136/ jnnp-2020-324927).
\end{abstract}

For numbered affiliations see end of article.

\section{Correspondence to} Dr Richard J Perry, Stroke Research Centre, UCL Queen Square Institute of Neurology, Queen Square, London WC1N 3BG, UK; richard.perry2@nhs. net

Received 19 August 2020 Revised 14 October 2020 Accepted 15 October 2020 Published Online First 5 November 2020

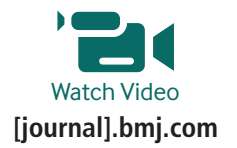

Check for updates

(C) Author(s) (or their employer(s)) 2021. No commercial re-use. See rights and permissions. Published by BMJ.

To cite: Perry RJ, Smith CJ, Roffe C, et al. J Neurol Neurosurg Psychiatry 2021:92:242-248.

\section{ABSTRACT}

Objective We set out to determine which

characteristics and outcomes of stroke are associated with COVID-19.

Methods This case-control study included patients admitted with stroke to 13 hospitals in England and Scotland between 9 March and 5 July 2020. We collected data on 86 strokes (81 ischaemic strokes and 5 intracerebral haemorrhages) in patients with evidence of COVID-19 at the time of stroke onset (cases). They were compared with 1384 strokes (1193 ischaemic strokes and 191 intracerebral haemorrhages) in patients admitted during the same time period who never had evidence of COVID-19 (controls). In addition, the whole group of stroke admissions, including another 37 patients who appeared to have developed COVID-19 after their stroke, were included in two logistic regression analyses examining which features were independently associated with COVID-19 status and with inpatient mortality.

Results Cases with ischaemic stroke were more likely than ischaemic controls to occur in Asians (18.8\% vs $6.7 \%, p<0.0002)$, were more likely to involve multiple large vessel occlusions (17.9\% vs $8.1 \%, p<0.03)$, were more severe (median National Institutes of Health Stroke Scale score 8 vs $5, p<0.002$ ), were associated with higher D-dimer levels $(p<0.01)$ and were associated with more severe disability on discharge (median modified Rankin Scale score 4 vs 3, $p<0.0001$ ) and inpatient death (19.8\% vs 6.9\%, $p<0.0001)$. Recurrence of stroke during the patient's admission was rare in cases and controls $(2.3 \%$ vs $1.0 \%$, NS).

Conclusions Our data suggest that COVID-19 may be an important modifier of the onset, characteristics and outcome of acute ischaemic stroke.

\section{INTRODUCTION}

COVID-19 is mainly recognised as a viral pneumonia, with a dry cough, high fever, shortness of breath and loss of taste and smell as its characteristic features. ${ }^{1}$ However, the virus responsible for this illness, SARS-CoV-2, may influence the presentation of ischaemic stroke, ${ }^{2}$ although this conclusion is controversial ${ }^{3}$ because of a lack of high quality evidence. In particular, the largest studies have compared patients with COVID-19 associated stroke with historical controls, ${ }^{4} 5$ whose strokes tend to be milder than those seen in contemporaneous controls, ${ }^{67}$ resulting in a bias towards overestimating the influence of COVID-19 on stroke severity and any other parameters correlated with severity.

Our objectives were to determine whether COVID-19 is associated with: a different demographic group; a higher proportion of ischaemic strokes; higher D-dimer values ${ }^{7}$ in ischaemic strokes, as may be expected of a SARS-CoV-2 related hypercoagulable state ${ }^{8}$; a different distribution of stroke mechanisms, possibly with a predominance of large vessel occlusions (LVOs) ${ }^{9}{ }^{10}$; more severe strokes with worse outcomes, including a higher inpatient mortality ${ }^{47}$; a higher rate of early recurrence of stroke; and a delay between the onset of symptoms of infection and of stroke. ${ }^{7}$ To address these objectives, we analysed data from a multicentre case-control study of strokes in patients admitted to 13 stroke services in England and Scotland during the 2020 COVID-19 pandemic.

\section{METHODS}

Study design and participants

SETICOS (Service Evaluation of The Impact of COVID-19 On Stroke) is an ongoing project in 13 stroke centres across England and Scotland (online supplemental table S1). Within this project, a casecontrol study was designed and reported according to STROBE guidelines. ${ }^{11}$ Approval was given by the clinical governance department in each hospital. The Health Research Authority confirmed that patient consent was not required for acquiring these surveillance data. There was a combination of retrospective and prospective collection. The study protocol was distributed to partner sites on 8 April 2020 and the first case report form was received on 14 April 2020. Estimated premorbid modified Rankin Scale (mRS) score and CT angiogram result were added to the list of fields on 29 April 2020, and our definition of recurrent stroke (see below) was clarified in data queries after data collection was complete. 
Anonymised case report forms were completed for patients admitted with a clinical diagnosis of stroke according to WHO criteria. $^{12}$ Patients with subarachnoid haemorrhage were excluded. We anticipated that most centres would not be able to collect data on consecutive stroke admissions throughout the whole study period, so centres were asked to prioritise weeks during which patients with COVID-19 were admitted, and for any such week (Monday to Sunday) to include all strokes, regardless of SARS-CoV-2 status.

Clinical data were extracted from discharge summaries or clinical notes, blood results were taken from electronic results systems and CT and MR angiogram data were obtained from radiology reports. Disability on discharge was determined using the $\mathrm{mRS}^{13}$ which varies from 0 (no symptoms) to 6 (death). For ischaemic strokes, the TOAST (trial of ORG 10172 in acute stroke treatment) classification ${ }^{14}$ was either taken from the discharge summary or was inferred from the clinical team's documented assessment of likely stroke aetiology. TOAST categories 'other determined aetiology' and 'undetermined aetiology' were combined, as the choice between these two subgroups would depend on whether the clinician regarded COVID-19 as a 'determined' cause of stroke. Data were checked centrally for omissions and inconsistencies, and data queries were submitted to local centres until they were resolved.

\section{Defining cases (strokes in patients with SARS-CoV-2 at the time of stroke onset) and controls}

For our SARS-CoV-2 positive 'case' group, we included all strokes in patients who tested positive within 4 days of admission (or within 4 days of their stroke for inpatient strokes), even if they were negative on their first test, because reverse transcriptasepolymerase chain reaction (RT-PCR) on respiratory samples has a low sensitivity for SARS-CoV-2. ${ }^{15}$ These patients would be very unlikely to have acquired the infection in hospital and turned PCR positive within such a short time. ${ }^{16}$ We also included strokes in patients who already had clinical features suspicious of COVID-19 at the time of admission and were found to be SARS-CoV-2 positive at any point during the first 10 days of admission. The 'control' group consisted of patients who were either consistently SARS-CoV-2 negative or were never tested because they did not show symptoms or signs of COVID-19. Patients who were SARS-CoV-2 positive at some point during their admission but did not satisfy the criteria to be counted in the case group were excluded from the case-control study but were included in the logistic regression analyses.

\section{Definition of recurrent stroke}

Recurrent stroke was defined as any new stroke occurring with an onset separate from that of the index stroke. If a recurrent stroke occurred within 21 days of the index stroke, and was in the same vascular territory, then it was only included if it additionally fulfilled at least one of the following two criteria: (1) there was a new area of acute infarction on neuroimaging or (2) there was a new intracerebral haemorrhage anatomically separate from the infarct or haemorrhage of the index stroke.

\section{Statistical methods}

Sample size was determined pragmatically by the data collection that was feasible in each centre during the challenging circumstances of the COVID-19 pandemic, aiming for at least 500 patients in total and at least 80 cases, sufficient to allow for logistic regression analyses. ${ }^{17}$ Categorical variables were compared between cases and controls using the $\chi^{2}$ test, except for stroke recurrence rates where Fisher's exact test was used because the expected rate of recurrent stroke in cases was fewer than five patients. D-dimer results were $\log _{10}$ transformed, resulting in distributions which approximated normal distributions, and then compared using the Student's t test (for a difference in the means) and an F test (for a difference in the variances). Other continuous variables were compared using the Mann-Whitney U test.

Binary logistic regression (Newton's method), using demographics, vascular risk factors and stroke characteristics that differed between the cases and controls (threshold $\mathrm{p}<0.2$ ), was used to establish which of these variables were independently associated with COVID-19 at onset. A further binary logistic regression analysis examined whether COVID-19 at onset was independently associated with inpatient mortality, using known predictors of early mortality ${ }^{18}$ as covariates of no interest. Where the admission National Institutes of Health Stroke Scale (NIHSS) score or estimated premorbid mRS score was not recorded, the median value for that covariate was entered in place of the missing data and a dummy covariate of no interest was added to the model, assigned a value of 1 for patients where the data point was missing and 0 for all other patients.

\section{RESULTS}

\section{Data included in the study}

We collected data from 1507 stroke admissions from the week commencing 9 March to the week commencing 29 June, from 13 stroke centres distributed across England and Scotland (online supplemental table S1). Early in the study period, patients were mainly tested for SARS-CoV-2 if there was clinical suspicion of COVID-19. Between the weeks commencing 9 March and 11 May, however, the proportion of asymptomatic patients tested increased progressively from $10.3 \%$ to $93.5 \%$, and then remained at a mean of $95.3 \%$ for the rest of the study.

Among the 123 strokes in patients who were SARS-CoV-2 positive at some point, 86 occurred in patients who had evidence of the infection at the time of stroke onset and were defined as cases (see methods). Nine of these (10.5\%) had no clinical features of COVID-19 during their admission. One case was in a patient who had also been admitted with a previous control stroke (without COVID-19) earlier during the study period. Thirty-six patients with COVID-19 were excluded from the casecontrol comparisons because they appeared to have contracted the infection after their stroke (see online supplemental table S2 for their characteristics). One further case with intracerebral haemorrhage was excluded because the date of stroke onset could not be estimated. The control group consisted of 1384 strokes in 1377 patients. For 823 of these strokes, the patient was negative on PCR $(59.5 \%)$ and in 561 strokes, the patient was not tested because they never had clinical evidence of COVID-19 (40.5\%). All 1507 strokes were included in logistic regression analyses.

\section{Demographics and stroke characteristics}

Ethnicity was recorded in $86.0 \%$ of cases and in $77.7 \%$ of controls. The proportion of Asian patients among cases (17.6\%) was more than twice that seen in the controls $(7.3 \%, \mathrm{p}<0.002$, table 1), a disparity entirely attributable to the difference found in the ischaemic stroke group ( $18.8 \%$ vs $6.7 \%, \mathrm{p}<0.0002$, table 2$)$. There was a correspondingly lower proportion of White patients among cases (table 1 and online supplemental figure S1). There was no significant difference in the proportion of Black patients between the two groups. Age and sex also did not differ between the two groups (online supplemental figure S2 and table 1). 
Table 1 Stroke characteristics in cases (with evidence of COVID-19 at stroke onset) and controls (with no evidence of COVID-19 at any time), including ischaemic and haemorrhagic strokes

\begin{tabular}{|c|c|c|c|}
\hline & Cases & Controls & $P$ value \\
\hline All strokes $(n)$ & 86 & 1384 & \\
\hline Age (years) (median (IQR)) & $74.5(67-84)$ & $73(61-82)$ & NS \\
\hline Men (n (\%)) & $47(54.7)$ & $731(52.8)$ & NS \\
\hline Ischaemic (n (\%)) & $81(94.2)$ & $1193(86.2)$ & 0.03 \\
\hline Ethnicity (n) & 74 & 1076 & \\
\hline White $(\mathrm{n}(\%))$ & $53(71.6)$ & $886(82.3)$ & 0.02 \\
\hline Black (n (\%)) & $7(9.5)$ & $98(9.1)$ & NS \\
\hline Asian $(\mathrm{n}(\%))$ & $13(17.6)$ & $79(7.3)$ & 0.002 \\
\hline Mixed/other $(n(\%))$ & $1(1.4)$ & $13(1.2)$ & NS \\
\hline Disability prior to stroke $(n)$ & 84 & 1369 & \\
\hline Premorbid mRS score (median (IQR)) & $1(0-3)$ & $0(0-2)$ & 0.01 \\
\hline Admission stroke severity (n) & 74 & 1336 & \\
\hline NIHSS score (median (IQR)) & $8(3.25-17)$ & $5(2-13)$ & 0.01 \\
\hline Respiratory support during admission ( $\mathrm{n}$ ) & 86 & 1370 & \\
\hline None required (n (\%)) & $41(47.7)$ & $1236(90.2)$ & $<0.00001$ \\
\hline Oxygen by nasal prongs $(\mathrm{n}(\%))$ & $22(25.6)$ & $66(4.8)$ & $<0.00001$ \\
\hline Oxygen by mask (n (\%)) & $15(17.4)$ & $42(3.1)$ & $<0.00001$ \\
\hline Non-invasive ventilation ( $\mathrm{n}(\%))$ & $1(1.2)$ & $6(0.4)$ & NS \\
\hline Intubation and ventilation (n (\%)) & $7(8.1)$ & $20(1.5)$ & $<0.00001$ \\
\hline Outcome measures (n) & 86 & 1384 & \\
\hline Length of stay (days) (median (IQR)) & $7(3-17)$ & $3(2-8)$ & $<0.00001$ \\
\hline mRS on discharge (median (IQR)) & $4(2-5)$ & $3(1-4)$ & 0.0004 \\
\hline Death during admission $(\mathrm{n}(\%))$ & $17(19.8)$ & $133(9.6)$ & 0.01 \\
\hline Recurrence during admission ( $\mathrm{n}(\%))$ & $2(2.3)$ & $14(1.0)$ & NS \\
\hline
\end{tabular}

mRS, modified Rankin scale; NIHSS, National Institutes of Health Stroke Scale.

There was a higher proportion of ischaemic strokes in the cases $(94.2 \%)$ compared with the controls $(86.2 \%, \mathrm{p}=0.03)$. Among ischaemic strokes that were classified according to TOAST criteria, ${ }^{14}$ there were no significant differences in the proportions of stroke attributed to large vessel atherosclerosis, cardioembolism or small vessel disease (table 2).

\section{D-dimers}

In patients with ischaemic stroke, D-dimers were measured in $23 / 81(28.4 \%)$ cases and in $177 / 1193(14.8 \%)$ controls; the distributions are shown on a $\log _{10}$ scale in figure 1 . Treating the two distributions in figure 1 as normal distributions of potentially differing variance, the cases had a significantly higher mean $\log _{10} \mathrm{D}$-dimer (3.4) compared with controls $(3.0, \mathrm{p}<0.01)$, and also a higher variance (SD 0.83 vs $0.63, p<0.03$ ), indicating a broader distribution of $\mathrm{D}$-dimers in cases rather than a rightward shift of the whole curve.

\section{Timing of COVID-19 and stroke onset}

In the 44 cases with ischaemic stroke in whom both dates were recorded, the onset of the COVID-19 relevant symptoms of fever, cough or dyspnoea occurred a median of 6 days before stroke onset (shown as -6 in table 2). For the three cases with intracerebral haemorrhage who had both dates recorded, COVID-19 symptoms occurred a median of 4 days after stroke onset, and although the numbers are small, the difference between these two medians was significant $(\mathrm{p}<0.005,2$-tailed test).

\section{Stroke severity on admission}

Figure 2 shows the distributions of ischaemic stroke severities on admission for cases and controls with ischaemic stroke, measured using the NIHSS. Ischaemic strokes were significantly more severe in the cases (median NIHSS score $=8$ ) than in the controls (median NIHSS score $=5, \mathrm{p}<0.002$ ). In patients with intracerebral haemorrhage, however, we did not find any significant difference in stroke severity between the two groups (median NIHSS scores $=9$ and 10 , table 2 ).

\section{Intracranial large vessel occlusion}

Among patients with ischaemic stroke, a CT or MR angiogram was available in $39 / 81$ cases (48.1\%) and in 627/1193 controls $(52.6 \%)$. The proportion of patients with at least one intracranial LVO was not significantly different between cases and controls, whether expressed as a proportion of the patients who had this imaging done $(41.0 \%$ vs $42.3 \%)$ or as a proportion of the whole stroke group (19.7\% vs $22.2 \%)$. However, of the patients who had CT or MR angiography, the proportion of cases with multiple intracranial LVOs $(17.9 \%)$ was more than twice that seen in controls $(8.1 \%, \mathrm{p}<0.03$; figure 3$)$.

\section{Features independently associated with COVID-19 status}

We used binary logistic regression to assess which demographics, vascular risk factors and stroke characteristics recorded on admission were independently associated with COVID-19 at stroke onset. Asian ethnicity and multiple intracranial LVOs on $\mathrm{CT}$ or MR angiography were independently associated with COVID-19 at stroke onset, while premorbid mRS narrowly missed statistical significance (table 3). In a sensitivity analysis, the pattern of results was not significantly affected by omission of the 37 patients who developed COVID-19 but did not fulfil the criteria for cases. 
Table 2 Comparisons between cases and controls, shown separately for ischaemic stroke and intracerebral haemorrhage

\begin{tabular}{|c|c|c|c|c|c|c|}
\hline & Ischaemic stroke & & & Intracerebral h & norrhage & \\
\hline & Cases & Controls & $P$ value & Cases & Controls & $P$ value \\
\hline All strokes (n) & 81 & 1193 & & 5 & 191 & \\
\hline Age (years) (median (IQR)) & $74(67-85)$ & $73(61-83)$ & NS & $76(67-80)$ & $73(61-80)$ & NS \\
\hline Men $(n(\%))$ & $44(54.3)$ & $633(53.1)$ & NS & $3(60.0)$ & $98(51.3)$ & NS \\
\hline Intravenous tPA (n (\%)) & $10(12.5)$ & $185(15.5)$ & NS & & & \\
\hline Thrombectomy (n (\%)) & $1(1.3)$ & $71(6.0)$ & NS & & & \\
\hline Ethnicity (n) & 69 & 939 & & 5 & 137 & \\
\hline White $(\mathrm{n}(\%))$ & $48(69.6)$ & $785(83.6)$ & 0.003 & $5(100.0)$ & $101(73.7)$ & NS \\
\hline Black (n (\%)) & $7(10.1)$ & $80(8.5)$ & NS & $0(0.0)$ & $18(13.1)$ & NS \\
\hline Asian $(\mathrm{n}(\%))$ & $13(18.8)$ & $63(6.7)$ & 0.0002 & $0(0.0)$ & $16(11.7)$ & NS \\
\hline Mixed/other (n (\%)) & $1(1.4)$ & $11(1.2)$ & NS & $0(0.0)$ & $2(1.5)$ & NS \\
\hline Admission stroke severity (n) & 70 & 1159 & & 4 & 177 & \\
\hline NIHSS (median (IQR)) & $8(3.25-11)$ & $5(2-11)$ & 0.002 & $9(3.25-14.25)$ & $10(4-20)$ & NS \\
\hline TOAST category $(\mathrm{n})$ & 72 & 1156 & & & & \\
\hline Large vessel atherosclerosis $(\mathrm{n}(\%))$ & $11(15.3)$ & $193(16.7)$ & NS & & & \\
\hline Cardioembolic (n (\%)) & $23(31.9)$ & $291(25.2)$ & NS & & & \\
\hline Small vessel $(\mathrm{n}(\%))$ & $18(25.0)$ & $227(19.6)$ & NS & & & \\
\hline Other (n (\%)) & $20(27.8)$ & $445(38.5)$ & NS & & & \\
\hline Fever/cough/SOB onset date ( $\mathrm{n}$ ) & 45 & & & 3 & & \\
\hline (Symptom onset) - (stroke onset) (days) (median (IQR)) & $-6(-14$ to 0$)$ & & & 4 (3 to 4$)$ & & \\
\hline $\mathrm{CT}$ or MR angiogram (n) & 39 & 627 & & & & \\
\hline No LVO (n (\%)) & $23(59.0)$ & $362(57.7)$ & NS & & & \\
\hline Single LVO (n (\%)) & $9(23.1)$ & $214(34.1)$ & NS & & & \\
\hline Multiple LVO (n (\%)) & $7(17.9)$ & $51(8.1)$ & 0.03 & & & \\
\hline Full blood count ( $n$ ) & 81 & 1173 & & 5 & 188 & \\
\hline NLR (median (IQR)) & $4.7(3.1-8.3)$ & $3.3(2.2-5.8)$ & 0.00005 & $5.2(3.7-8.9)$ & $3.9(2.2-7)$ & NS \\
\hline Platelets (median (IQR)) & $251(185-342)$ & 241 * (200.5-293) & NS & $273(230-275)$ & $232(185-293)$ & NS \\
\hline $\operatorname{CRP}(n)$ & 78 & 1051 & & 5 & 162 & \\
\hline CRP (mg/L) (median (IQR)) & $29.8(7.4-114.9)$ & $5.0(1.9-14.25)$ & $<0.00001$ & $2.9(1.6-5.2)$ & $5.0(2-13)$ & NS \\
\hline D-dimer (n) & 23 & 177 & & & & \\
\hline $\log _{10}$ D-dimer $(\mathrm{ng} / \mathrm{mL})($ mean $(\mathrm{SE}))$ & $3.4(0.20)$ & $3.0(0.04)$ & 0.01 & & & \\
\hline Outcome measures (n) & 81 & 1193 & & 5 & 191 & \\
\hline Length of stay (days) (median (IQR)) & $7(3-17)$ & $3(2-7)$ & $<0.00001$ & $9(7-11)$ & $5(2-10)$ & NS \\
\hline mRS on discharge (median (IQR)) & $4(2-5)$ & $3(1-4)$ & 0.00003 & $4(3-4)$ & $4(3-6)$ & NS \\
\hline Death during admission ( $\mathrm{n}(\%))$ & $16(19.8)$ & $82(6.9)$ & 0.00003 & $1(20)$ & $51(26.7)$ & NS \\
\hline Recurrence during admission (n (\%)) & $2(2.5)$ & $12(1.0)$ & NS & $0(0.0)$ & $2(1.0)$ & NS \\
\hline
\end{tabular}

${ }^{*}$ Omitting five patients in whom the platelets clumped.

CRP, C reactive protein; LVO, large vessel occlusion; mRS, modified Rankin scale; NIHSS, National Institutes of Health Stroke Scale; NLR, neutrophil to lymphocyte ratio; SOB, shortness of breath; TOAST, trial of ORG 10172 in acute stroke treatment; tPA, tissue plaminogen activator.

\section{Outcome from stroke}

Stroke recurrence during admission was very rare in cases $(2.3 \%)$ and controls (1.0\%), and with such small numbers it was not possible to demonstrate any significant difference between these groups, whether all strokes (table 1) or only ischaemic strokes (table 2) were included. The rates in cases and controls are in any case not directly comparable because cases had a longer median length of stay (table 1).

The proportion of ischaemic strokes resulting in death during admission was significantly higher in cases $(19.8 \%)$ than in controls $(6.9 \%, \mathrm{p}<0.00003$, table 2$)$, and the median $\mathrm{mRS}$ on discharge was higher in ischaemic cases (median mRS score $=4$ ) than in ischaemic controls (median mRS score $=3, p<0.00003$, figure 4). For intracerebral haemorrhages, we were unable to demonstrate any significant difference in disability or mortality between the 5 cases (median mRS score $=4$, mortality 20.0\%) and the 191 controls (median mRS score $=4$, mortality 26.7\%). COVID-19 at the onset of the stroke was independently associated with death during admission, even after correction for parameters known to be associated with early mortality ${ }^{18}$ (table 4).

\section{DISCUSSION}

This UK-based multicentre study provides a large case-control comparison, with contemporaneous controls, of strokes with and without COVID-19. Ischaemic strokes which were associated with COVID-19 at onset were: more likely to occur in Asian people; more likely to involve multiple LVOs; more severe; associated with higher D-dimer levels; and more likely to have a worse functional outcome or result in death. These findings add substantially to the previous smaller and methodologically limited studies, summarised in a recent systematic review, ${ }^{2}$ to confirm that COVID-19 has an important influence over the onset, characteristics and outcome of acute ischaemic stroke. 


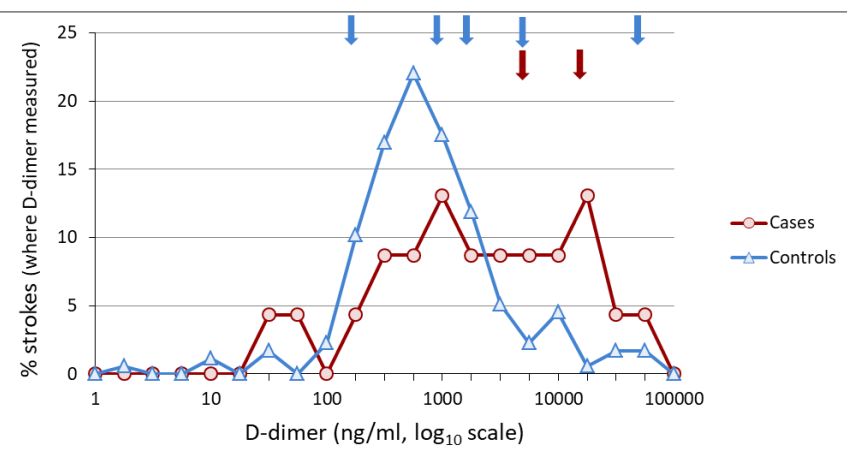

Figure 1 Distribution of D-dimers $(\mathrm{ng} / \mathrm{mL})$ in cases and controls with ischaemic stroke, plotted on a $\log _{10}$ scale. Bin width of $\log _{10} D$-dimer=0.25. The value shown on the $x$ axis is the lower value of each bin. The value on the $y$ axis is the percentage of D-dimer results falling within that range of values. Each arrow along the top row indicates one control who was anticoagulated for a deep vein thrombosis or pulmonary embolism during their admission; each arrow on the bottom row indicates a case who was anticoagulated for these indications.

Previous case series suggested that COVID-19 may be associated with a higher proportion of strokes with $\mathrm{LVOs}^{2910}$ and this conclusion was confirmed in a single case-control study. ${ }^{19}$ In our study, by contrast, the overall incidence of at least one LVO was the same in the case and control groups. The proportion of cases with multiple LVOs, however, was more than twice that seen in the control group.

LVO in COVID-19 may be a direct manifestation of a SARS-CoV-2 related hypercoagulable state, in which D-dimers are elevated. ${ }^{79}$ In our study, not only was the mean $\log _{10}$ $\mathrm{D}$-dimer higher in cases than in controls, but also the variance of $\log _{10}$ D-dimers was higher in cases, suggesting greater heterogeneity in this group; one potential explanation would be the presence of a subgroup with higher D-dimers associated with a SARS-CoV-2 related coagulopathy.

Our finding that ischaemic stroke associated with COVID-19 is more severe than in patients without COVID-19 is consistent with a small case-control study from New York, which reported more severe strokes in 32 patients with COVID-19 (median NIHSS score $=19$ ) than in 46 contemporaneous control patients without the infection (median NIHSS score $=8){ }^{7}$ However, strokes in their contemporaneous control group were

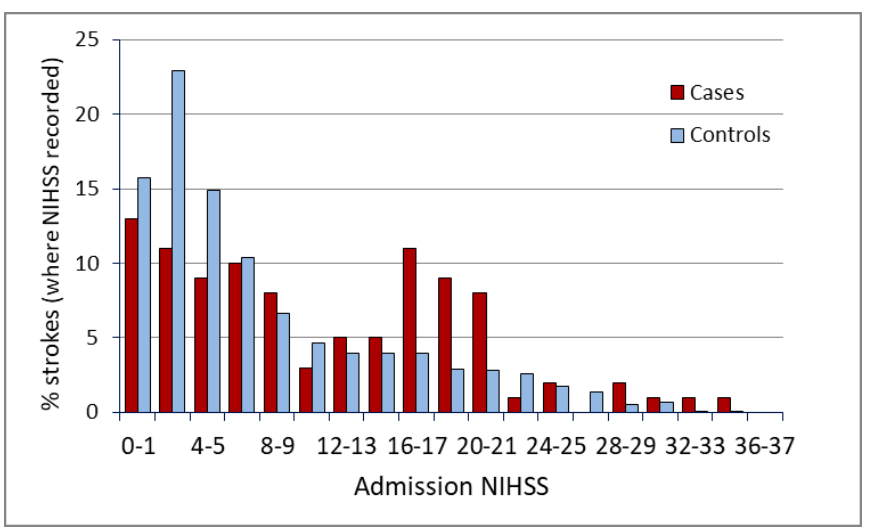

Figure 2 Distribution of National Institutes of Health Stroke Scale (NIHSS) scores in cases and controls with ischaemic stroke. For each NIHSS range, the frequency of cases is shown as a percentage of the cases in which the NIHSS was measured, and similarly for controls. Bin width $=2$.

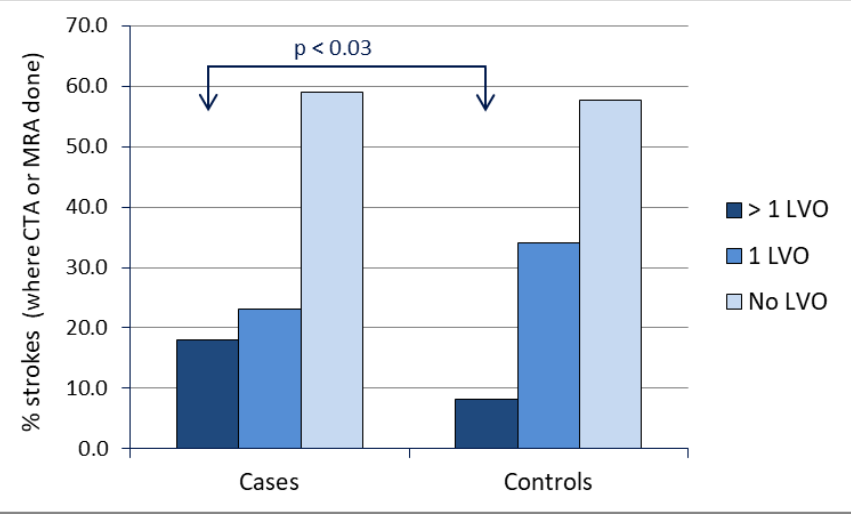

Figure 3 Relationship between CT angiogram (CTA) or MR angiogram (MRA) findings and COVID-19 status in patients with ischaemic stroke. For cases and controls, shown are the proportions of those scanned in whom more than one intracranial large vessel occlusion (LVO) was reported, only one LVO was reported or no LVO was reported.

considerably more severe than they were in their historical controls (median NIHSS score $=3$ ). The difference presumably reflects a strong tendency for patients with minor stroke to stay away from hospital during the pandemic, or for doctors to avoid admitting them. Although we have observed the same effect in the UK, ${ }^{6}$ the degree of exclusion of minor strokes during the pandemic appears to have been less pronounced in our study (control group median NIHSS score $=5$ ). This disparity may reflect differences in public behaviour during the pandemic in different populations, or differences in admission strategies between the group of UK hospitals included in our study and the private healthcare organisation (NYU Langone Health) in the New York study.

The shift towards greater stroke severity in COVID-19 negative patients during the pandemic ${ }^{6}$ means that registry studies comparing cases of COVID-19 associated stroke with historical controls $^{45}$ are biased towards overestimating any influence of COVID-19 on stroke severity, and other correlated parameters, such as the incidence of LVOs and inpatient mortality rate, because historical controls have milder strokes than contemporaneous controls. ${ }^{7}$ We have avoided this bias by comparing our COVID-19 cases with contemporaneous controls. Even so, outcomes were still worse in cases than controls in our study, and in particular the inpatient mortality rate in our cases was significantly higher, a finding that remained after correction for other known risk factors for early inpatient mortality. ${ }^{18}$

Table 3 Binary logistic regression analysis to explore the association of demographics, vascular risks factors and stroke characteristics on admission with COVID-19 at the time of stroke. Covariates are shown in order of statistical significance

\begin{tabular}{lllll}
\hline & OR & $95 \% \mathrm{Cl}$ & & P value \\
\hline Asian & 2.70 & 1.38 & 5.30 & 0.004 \\
More than 1 LVO & 2.62 & 1.11 & 6.15 & 0.03 \\
Premorbid mRS score & 1.16 & 0.99 & 1.35 & 0.07 \\
Current smoker & 0.50 & 0.21 & 1.19 & 0.12 \\
Admission NIHSS score & 1.02 & 0.99 & 1.05 & 0.14 \\
Type 2 diabetes & 1.28 & 0.77 & 2.12 & 0.34
\end{tabular}

$95 \% \mathrm{Cl}, 95 \%$ confidence interval; LVO, large vessel occlusion; mRS, modified Rankin Scale; NIHSS, National Institutes of Health Stroke Scale; OR, odds ratio. 


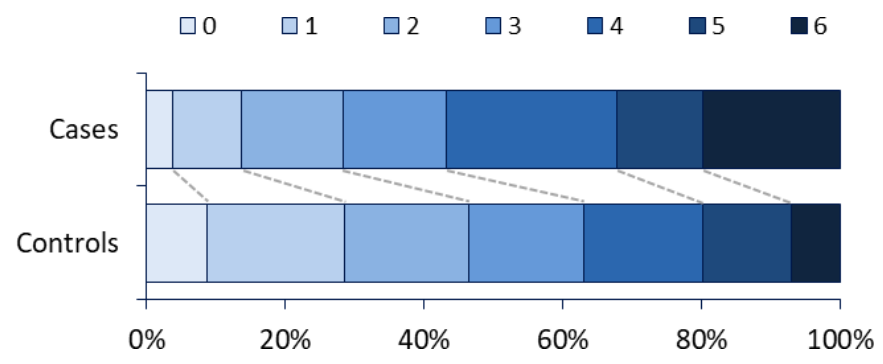

Figure 4 Distribution of modified Rankin Scale (mRS) scores on discharge in cases and controls with ischaemic stroke. The mRS score indicates the degree of disability: for example, $0=$ no symptoms,

$1=$ symptoms without disability, $5=$ severe disability and $6=$ death during admission.

We found no evidence to support previous suggestions that SARS-CoV-2 infection may be associated with a younger age a $^{710}$ or male $\operatorname{sex}^{7}$ in stroke patients. In the case-control study from New York, ${ }^{7}$ by contrast, patients with COVID-19 associated strokes were younger (median 63 years) than controls (70 years). Most of their patients with COVID-19 associated stroke presented with COVID-19 as their primary diagnosis, so a likely explanation for this disparity is that, in their study, older patients with COVID-19 as their primary diagnosis may have been less likely to present and be admitted to hospital during the pandemic than younger patients.

There was a median delay of 6 days from the onset of COVID-19 symptoms to the onset of ischaemic stroke, perhaps corresponding to the reported delay between COVID-19 onset and the development of a hypercoagulable state. ${ }^{8}$ However, COVID-19 does not appear to influence stroke solely through a single mechanism; no single aetiological category of ischaemic stroke seems to have been more strongly associated with COVID-19 infection than the others. We suggest that COVID-19 may provoke the onset of an ischaemic stroke through a variety of thrombotic and inflammatory mechanisms, promoting generation of thrombus in the heart ${ }^{20}$ or large vessels ${ }^{1021}$ or via small vessel occlusion. ${ }^{22}$ Which of these mechanisms manifests in a given patient may be determined by that individual's conventional vascular risk factors, such as atrial fibrillation, large vessel atheroma, hypertension or type 2 diabetes mellitus.

Strengths of our study are that we included patients from 13 centres across the UK, so our results may be more representative of the overall stroke population than existing studies from a single hospital system or city. The use of contemporaneous controls allowed us to draw conclusions about the influence

Table 4 Binary logistic regression analysis demonstrating that COVID-19 at stroke onset was associated with inpatient mortality, even after correction for other clinical features known to be predictors of early mortality ${ }^{18}$

\begin{tabular}{lllll}
\hline & OR & $95 \% \mathrm{Cl}$ & & P value \\
\hline Admission NIHSS score & 1.16 & 1.13 & 1.19 & $<0.00001$ \\
\hline Intracerebral haemorrhage & 3.19 & 1.99 & 5.11 & $<0.00001$ \\
\hline Age (per year) & 1.03 & 1.01 & 1.04 & 0.002 \\
\hline Ischaemic heart disease & 1.92 & 1.21 & 3.02 & 0.005 \\
\hline COVID-19 at stroke onset & 2.11 & 1.08 & 4.13 & 0.03 \\
\hline Premorbid mRS score & 1.12 & 0.98 & 1.30 & 0.11 \\
Type 2 diabetes mellitus & 1.37 & 0.86 & 2.19 & 0.19 \\
\hline Small vessel ischaemic stroke & 0.64 & 0.29 & 1.42 & 0.27 \\
\hline
\end{tabular}

mRS, modified Rankin Scale; NIHSS, National Institutes of Health Stroke Scale. of COVID-19 on stroke severity, mortality and the incidence of LVO, inferences that cannot be made in studies comparing registry data with historical controls. In addition, the study was conducted according to a protocol agreed on early in the pandemic, before the frequency of cases in the UK had reached its peak.

The main limitation of this study is that we were only able to report investigations done as part of routine clinical care. Some reports or tests were not available in all patients, which could introduce reporting or indication biases. For example, if there were a higher rate of reporting of ethnicity in cases compared with controls only in Asians, this reporting bias could in theory have exaggerated the association between Asian patients and COVID-19, although such a specific reporting bias seems implausible.

D-dimers were measured in a higher proportion of cases (28.4\%) than controls (14.8\%), suggesting that the criteria for measuring them may have been narrower in the control group. Assuming that narrower test criteria select patients with higher D-dimers, there may have been a bias towards higher D-dimers in controls, resulting in an underestimate of the difference in D-dimers between the two groups. Although an indication bias could also have operated in the selection of patients for CT angiography, reassuringly we found no evidence of any bias towards performing this imaging in patients with COVID-19.

Not all control patients were tested for SARS-CoV-2, so this group may have included patients with asymptomatic infection. In addition, we were reliant on SARS-CoV-2 RT-PCR results from respiratory swabs for COVID-19 diagnosis, and this test has a very poor sensitivity. ${ }^{16}$ However, the large size of our control group will have minimised the influence of false negative COVID-19 results over this group.

If patients with minor stroke were more likely to attend if they had symptoms of COVID-19, then this effect could have reduced the median NIHSS score in cases, causing us to underestimate the difference in stroke severity between cases and controls. Finally, some COVID-19 associated strokes may have been missed in patients in whom clinical assessment was hampered by very severe COVID-19 infection.

Our study provides the most compelling evidence yet that COVID-19 associated ischaemic strokes are more severe and more likely to result in severe disability or death, although the outlook is not quite as bleak as previous studies have suggested. ${ }^{78}$ Our results suggest the following recommendations for management of stroke patients during the ongoing COVID-19 pandemic.

If at any point a stroke centre is not routinely testing all stroke admissions for SARS-CoV-2, patients presenting with ischaemic stroke and very elevated D-dimers with no other explanation should be considered for testing, even if the clinical suspicion of COVID-19 is otherwise low. Criteria for requesting CT angiography in stroke patients may in the future need to take account of their COVID-19 status, because the finding of multiple LVOs may mean that a specific management strategy is required, such as a different antithrombotic agent. On the other hand, in most patients with COVID-19 associated ischaemic stroke, very early anticoagulation is probably not warranted as a strategy to prevent inpatient stroke recurrence, as this outcome is too uncommon to justify the increased risk of secondary haemorrhage.

\section{Author affiliations}

${ }^{1}$ Comprehensive Stroke Service, Box 119, National Hospital for Neurology and Neurosurgery, London, UK 
${ }^{2}$ Stroke Research Centre, UCL Queen Square Institute of Neurology, London, UK ${ }^{3}$ Manchester Centre for Clinical Neurosciences, Manchester Academic Health Science Centre, Salford, UK

${ }^{4}$ Division of Cardiovascular Sciences, Lydia Becker Institute of Immunology and Inflammation, University of Manchester, Manchester, UK

${ }^{5}$ Faculty of Medicine and Health Sciences, Keele University, Keele, UK

${ }^{6}$ Stroke Service, University Hospitals of North Midlands NHS Trust, Stoke-on-Trent, UK ${ }^{7}$ Department of Stroke Medicine, Pennine Acute Hospitals NHS Trust, Greater Manchester, UK

${ }^{8}$ Department of Stroke Medicine, University Hospital Southampton NHS Foundation Trust, Southampton, UK

${ }^{9}$ Department of Neurology, University Hospitals Birmingham NHS Foundation Trust, Birmingham, UK

${ }^{10}$ Department of Stroke Medicine, Newcastle upon Tyne Hospitals NHS Foundation Trust, Newcastle upon Tyne, UK

${ }^{11}$ Department of Neurology, The Leeds Teaching Hospitals NHS Trust, Leeds, UK

${ }^{12}$ Institute of Cardiovascular and Medical Sciences, University of Glasgow, Glasgow, UK

${ }^{13}$ Stroke Service, Glasgow Royal Infirmary, Glasgow, UK

${ }^{14}$ Department of Neurology, King's College Hospital NHS Foundation Trust, London, UK

${ }^{15}$ Department of Neurology, St George's University Hospitals NHS Foundation Trust, London, UK

${ }^{16}$ Stroke Centre, Imperial College Healthcare NHS Trust, London, UK

Twitter Craig J Smith @cjsmithdr, Terence J Quinn @DrTerryQuinn and Isobel H Marks@issymarks

Acknowledgements The authors would like to thank the whole team of SETICOS collaborators (listed in the supplemental material) who undertook their tasks largely on a voluntary basis during the exceptionally challenging time of the COVID-19 pandemic. We would also like to thank the two anonymous reviewers who, on behalf of this journal, gave us important detailed feedback on an earlier version of this manuscript. This work was undertaken at UCL Hospitals/UCL, which receives a proportion of funding from the Department of Health's National Institute for Health Research (NIHR) Biomedical Research Centre's funding scheme.

Contributors RJP, RS and DJW conceived the study. RJP wrote and distributed the protocol, designed the case report form and registration log, recruited centres to the study, received the case report forms and uploaded them into the database, performed statistical analyses and wrote the manuscript. RJP, CJS, CR, RS and DJW were the members of the Core Writing Group and critically reviewed the protocol, case report form and manuscript. CJS, CR, SN, RM, MW, AD, AH, TJQ, SA, LZ, AB and $S B$ obtained approval for the study at their respective sites and took overall responsibility for the submission of accurate data. SN, RM, MW, TJQ, UA, NP, PF, FM, $A B$ and IHM extracted data from clinical records and completed case report forms, working alongside collaborators listed in the supplemental materials. All authors critically reviewed the manuscript and approved the final version.

Funding The authors have not declared a specific grant for this research from any funding agency in the public, commercial or not-for-profit sectors.

Competing interests CJS has received honoraria from Bayer, Sanofi and Pfizer, not related to the work presented here. DJW has received personal fees from Bayer, Alnylam and Portola, not related to the work presented here.

Patient consent for publication Not required.

Ethics approval Approval for the study was given by the clinical governance department in each hospital. The Health Research Authority confirmed that patient consent was not required for acquiring these surveillance data.

Provenance and peer review Not commissioned; externally peer reviewed.

Data availability statement Data are available on reasonable request, subject to restrictions imposed by patient confidentiality.

Supplemental material This content has been supplied by the author(s). It has not been vetted by BMJ Publishing Group Limited (BMJ) and may not have been peer-reviewed. Any opinions or recommendations discussed are solely those of the author(s) and are not endorsed by BMJ. BMJ disclaims all liability and responsibility arising from any reliance placed on the content. Where the content includes any translated material, BMJ does not warrant the accuracy and reliability of the translations (including but not limited to local regulations, clinical guidelines, terminology, drug names and drug dosages), and is not responsible for any error and/or omissions arising from translation and adaptation or otherwise.

\section{ORCID iDs}

Richard J Perry http://orcid.org/0000-0002-4536-9018

Craig J Smith http://orcid.org/0000-0002-9078-9919

Christine Roffe http://orcid.org/0000-0002-5259-6649

Robert Simister http://orcid.org/0000-0003-0578-6711

Richard Marigold http://orcid.org/0000-0003-1924-1616

Terence J Quinn http://orcid.org/0000-0003-1401-0181

Soma Banerjee http://orcid.org/0000-0003-2466-2909

David J Werring http://orcid.org/0000-0003-2074-1861

\section{REFERENCES}

1 Docherty AB, Harrison EM, Green CA, et al. Features of 20133 UK patients in hospital with covid-19 using the ISARIC WHO Clinical Characterisation Protocol: prospective observational cohort study. BMJ 2020:m1985.

2 Tan Y-K, Goh C, Leow AST, et al. COVID 19 and ischemic stroke: a systematic review. J Thromb Thrombolysis 2020;50:587-95.

3 Morelli N, Spallazzi M, Rota E, et al. Occam's razor, stroke, and COVID-19. AJNR Am J Neuroradiol 2020;41:E62-3

4 Ntaios G, Michel P, Georgiopoulos G, et al. Characteristics and outcomes in patients with COVID-19 and acute ischemic stroke. Stroke 2020;51.

5 Katz JM, Libman RB, Wang JJ, et al. Cerebrovascular complications of COVID-19. Stroke 2020;51:e227-31.

6 Perry R, Banaras A, Werring DJ, et al. What has caused the fall in stroke admissions during the COVID-19 pandemic? J Neurol 2020. doi:10.1007/s00415-020-10030-2. [Epub ahead of print: 29 Jun 2020].

7 Yaghi S, Ishida K, Torres J, et al. SARS-CoV-2 and stroke in a new York healthcare system. Stroke 2020;51:2002-11.

8 Tang N, Li D, Wang X, et al. Abnormal coagulation parameters are associated with poor prognosis in patients with novel coronavirus pneumonia. J Thromb Haemost 2020:18:844-7.

9 Beyrouti R, Adams ME, Benjamin L, et al. Characteristics of ischaemic stroke associated with COVID-19. J Neurol Neurosurg Psychiatry 2020;91:889-91.

10 Oxley TJ, Mocco J, Majidi S, et al. Large-vessel stroke as a presenting feature of Covid-19 in the young. N Engl J Med 2020;382:e60.

11 Vandenbroucke JP, von Elm E, Altman DG, et al. Strengthening the reporting of observational studies in epidemiology (STROBE): explanation and elaboration. PLOS Med 2007;4:e297.

12 Aho $\mathrm{K}$, Harmsen $\mathrm{P}$, Hatano $\mathrm{S}$, et al. Cerebrovascular disease in the community: results of a WHO collaborative study. Bull World Health Organ 1980;58:113-30.

13 van Swieten JC, Koudstaal PJ, Visser MC, et al. Interobserver agreement for the assessment of handicap in stroke patients. Stroke 1988;19:604-7.

14 Adams HP, Bendixen BH, Kappelle LJ, et al. Classification of subtype of acute ischemic stroke. Definitions for use in a multicenter clinical trial. TOAST. trial of ORG 10172 in acute stroke treatment. Stroke 1993;24:35-41.

15 Woloshin S, Patel N, Kesselheim AS. False negative tests for SARS-CoV-2 infection challenges and implications. N Eng/ J Med 2020;383:e38.

16 Kucirka LM, Lauer SA, Laeyendecker 0 , et al. Variation in false-negative rate of reverse transcriptase polymerase chain reaction-based SARS-CoV-2 tests by time since exposure. Ann Intern Med 2020;173:262-7.

17 Bujang MA, Sa'at N, Sidik TMITAB, et al. Sample size guidelines for logistic regression from observational studies with large population: emphasis on the accuracy between statistics and parameters based on real life clinical data. Malays J Med Sci 2018;25:122-30

18 Gattringer T, Posekany A, Niederkorn K, et al. Predicting early mortality of acute ischemic stroke. Stroke 2019;50:349-56.

19 Kihira S, Schefflein J, Mahmoudi K, et al. Association of coronavirus disease (COVID-19) with large vessel occlusion strokes: a case-control study. Am J Roentgenol:1-6.

20 Ford JS, Holmes JF, Jones RF. Cardioembolic stroke in a patient with coronavirus disease of 2019 (COVID-19) myocarditis: a case report. Clin Pract Cases Emerg Med 2020:4:332-5

21 Mohamud AY, Griffith B, Rehman M, et al. Intraluminal carotid artery thrombus in COVID-19: another danger of cytokine storm? AJNR Am I Neuroradiol 2020:41:1677-82.

22 Nicholson P, Alshafai L, Krings T. Neuroimaging findings in patients with COVID-19. AJNR Am J Neuroradiol 2020:41:1380-3. 